\title{
LITERACIA EM SAÚDE: RESULTADOS OBTIDOS A PARTIR DE UMA AMOSTRA DE PESSOAS IDOSAS PORTUGUESAS
}

\author{
| Carla Serrão ${ }^{1}$; Sofia Veiga ${ }^{2}$; Isabel Vieira ${ }^{3} \mid$
}

\section{RESUMO}

CONTEXTO: O presente estudo insere-se no Projeto Literacia em Saúde: um desafio na e para a terceira idade, financiado pela Fundação Calouste Gulbenkian.

OBJECTIVO(S): Tem como intuito avaliar o grau de literacia em saúde de uma amostra de pessoas idosas, bem como conhecer a associação entre o grau de literacia em saúde e algumas características sociodemográficas dos participantes.

METOLODOGIA: Para tal foi realizada uma pesquisa de carácter quantitativo junto de 433 adultos com mais de 65 anos de idade, utilizando-se como instrumento o Newest Vital Sign (NVS).

RESULTADOS: Os resultados indicam que a grande maioria dos sujeitos participantes (80\%) evidenciou um nível de literacia em saúde baixo, o que significa que apenas $20 \%$ dos inquiridos serão capazes de interpretar e usar a informação escrita relacionada com a saúde de forma eficaz. O sexo, as habilitações literárias, a idade e o estado civil revelaram-se variáveis que afetam significativamente o grau de literacia em saúde dos participantes.

CONCLUSÕES: Os resultados obtidos apontam para a necessidade urgente de se potenciar a literacia em saúde na população idosa, em geral, e junto dos grupos de maior vulnerabilidade, em particular.

\section{PALAVRAS-CHAVE: Alfabetização em saúde; Idoso; Sexo; Escolaridade}

\section{RESUMEN}

"La alfabetización en salud: Resultados de una muestra de personas mayores Portugueses"

CONTEXTO: Este estudio se inscribe en el Proyecto de Alfabetización en Salud: un reto en y para las personas mayores, financiado por la Fundación Calouste Gulbenkian.

OBJETIVO: Tiene la intención de evaluar el grado alfabetización en salud de una muestra de personas mayores, así como conocer la asociación entre el grado de alfabetización en salud y las características sociodemográficas de los participantes.

MATERIAL Y MÉTODOS: Se llevó a cabo una investigación de carácter cuantitativo con 433 adultos mayores de 65 años de edad, utilizando como instrumento el Newest Vital Sign (NVS).

RESULTADOS: Los resultados indican que la gran mayoría de los sujetos participantes (80\%) mostró un nivel de alfabetización en salud bajo, lo que significa que sólo el $20 \%$ de los encuestados será capaz de interpretar y utilizar información escrita relacionada con salud de manera eficaz. El sexo, el nivel educativo, la edad y el estado civil resultaron variables que afectan de manera significativa el nivel de alfabetización en salud de los participantes.

CONCLUSIONES: Los resultados apuntan a la necesidad urgente de aumentar la alfabetización en salud en la población anciana en general, y entre los grupos más vulnerables, en particular.

\section{DESCRIPTORES: Alfabetização em saúde; Idoso; Sexo; Esco-} laridade

\begin{abstract}
"Health literacy: Results from a sample of Portuguese elderly people"

BACKGROUND: The present study integrates the Health Literacy Project: a challenge in and for the elderly people, funded by the Calouste Gulbenkian Foundation.

AIM: It has the intention to evaluate the degree of health literacy on a sample of elderly people, as well as knowing the association between this degree and sociodemographic characteristics of the participants.

MATERIAL AND METHODS: It was conducted a quantitative study with 433 adults over 65 years of age, using as instrument the Newest Vital Sign (NVS).

RESULTS: The results indicate that the majority of the participants (80\%) showed a level of low health literacy, meaning that only $20 \%$ of respondents will be able to interpret and use effectively written information related with health. Sex, educational attainment, age and marital status proved to be variables that affect significantly the level of health literacy of participants.

CONCLUSIONS: The results point to the urgent need to enhance health literacy in the elderly population, in general, and among the most vulnerable groups, in particular.
\end{abstract}

\section{KEYWORDS: Health literacy; Aged; Sex; Educational status}

Submetido em 29-09-2014

Aceite em 13-12-2014

1 Psicóloga Clínica; Professora Adjunta no Instituto Politécnico do Porto, Escola Superior de Educação, Unidade Técnico Científica de Psicologia, carlaserrao@ese.ipp.pt 2 Psicóloga; Professora Adjunta no Instituto Politécnico do Porto, Escola Superior de Educação, Unidade Técnico Científica de Psicologia, Porto, sofiaveiga@ese.ipp.pt 3 Educadora Social; Assistente no Instituto Politécnico do Porto, Escola Superior de Educação, Unidade Técnico Científica de Ciências da Educação, isabelvieira@ese.ipp.pt 


\section{INTRODUÇÃO}

De acordo com a definição desenvolvida em 2012 pelo European Health Literacy Consortium (WHO, 2013), a literacia em saúde está ligada à literacia e capacita as pessoas de conhecimentos, motivação e competências para aceder, compreender, avaliar e mobilizar informações relativas à saúde, a fim de fazerem julgamentos e tomarem decisões na sua vida quotidiana relativos a cuidados de saúde, prevenção de doenças e promoção da saúde para manter ou melhorar a qualidade de vida ao longo do ciclo vital. Uma inadequada literacia em saúde tem, como é óbvio, implicações várias na receção de cuidados de saúde, na medida em que os pacientes necessitam de saber ler rótulos medicinais, receitas e apontamentos médicos, entender instruções de autocuidados de saúde, etc., o que é motivo suficiente para associar baixa literacia em saúde com um pior nível de saúde (Speros, 2005). Além disso, tem-se também verificado que uma inadequada literacia em saúde se relaciona com um pior estado de saúde geral, físico e mental (Speros, 2005; Wolf, Gazmararian, \& Baker, 2005).

Os estudos desenvolvidos apontam que a prevalência de baixa literacia em saúde parece ser maior na população idosa, em indivíduos com baixo status social, baixo nível educacional e baixos recursos financeiros, bem como em pacientes com doenças crónicas e em doentes que recorrem aos serviços de saúde públicos (Manafo, \& Wong, 2012; Schillinger et al., 2002; Speros, 2005; Sudore et al., 2006; WHO, 2013). Relativamente às pessoas com mais de 65 anos, o estudo de Wolf et al. (2005) concluiu uma relação entre grau de literacia em saúde e função física e saúde mental. Ou seja, níveis mais baixos de literacia em saúde relacionam-se com uma pior função física e saúde mental; uma maior probabilidade de reportarem dificuldades em atividades instrumentais do quotidiano e atividades quotidianas; uma maior limitação na atividade devido a uma saúde física debilitada; menos realizações devido à frágil saúde física e um maior número de relatos de dor que interfere com a atividade quotidiana. Também Kim (2009) chegou a resultados semelhantes, concluindo que indivíduos com mais de 65 anos que apresentavam uma inadequada literacia em saúde evidenciavam níveis significativamente mais elevados de artrite e hipertensão, reportavam maiores limitações na atividade diária e uma pior saúde subjetiva, bem como níveis mais elevados de dor. Relativamente a algumas variáveis demográficas, diversos estudos concluíram que a literacia em saúde era marcadamente menor nos idosos com mais idade (Baker, Gazmararian, Sudano, \& Patterson, 2000) e menos escolarizados (Bennett, Chen, Soroui, \& White, 2009).
Todavia, no que concerne ao sexo, não se verifica concordância nos resultados obtidos em diferentes investigações, revelando uns que os homens apresentam uma maior literacia em saúde (Jovic-Vranes, BjegovicMikanovic, \& Marinkovic, 2009; Toçi et al., 2013), enquanto outros revelam o contrário (Sudore et al., 2006; Von Wagner, Knight, Steptoe, \& Wardle, 2007).

Não se encontraram estudos que investigassem a variável estado civil.

No sentido de reverter esta situação, a Organização Mundial de Saúde (WHO, 2013) apela para a necessidade de se tomarem um conjunto de medidas sensíveis ao sexo, à idade e às diferenças e diversidades educacionais e culturais (e.g., etnia, religião, língua e cultura de origem) das pessoas idosas.

\section{METODOLOGIA}

O estudo, de carácter quantitativo, integrou uma amostra de 433 adultos com mais de 65 anos de idade. Os participantes responderam ao Newest Vital Sign (NVS, Copyright (C) Pfizer Inc.) instrumento desenvolvido por Weiss et al. (2005), que avalia o grau de literacia em saúde.

Recorrendo a um rótulo nutricional de uma embalagem de gelado, o NVS contempla seis questões de resposta fechada que implicam competências de literacia variadas, incluindo competências de leitura e de matemática, nomeadamente a consulta e interpretação da informação disponibilizada (ex., "Suponha que é alérgico a (...). É seguro para si comer este gelado?"), assim como a realização de inferências e de cálculos mentais (ex. "Se habitualmente consumir 2500 calorias por dia, qua percentagem desse valor é que estará a consumir se comer uma porção de gelado?). As respostas são cotadas tendo por base critérios específicos e pré-definidos. O total é obtido através da soma das respostas corretas.

A aplicação do NVS decorreu individualmente, sendo possível fazê-lo de três modos distintos: autoadministrado, em que, após a leitura das instruções, o participante lia e preenchia o questionário sem qualquer ajuda ( $n=198 ; 46.2 \%)$; assistido pelo observador, em que este lia as instruções e explicava o modo de preenchimento do questionário, mas era o sujeito idoso que lia as questões e assinalava as respostas $(n=26 ; 6.1 \%)$; administrado pelo observador, em que este lia as instruções, as questões e assinalava as respostas dadas pelo participante $(n=205 ; 47.8 \%)$. 
A amostra integrou sujeitos caucasianos, com idades compreendidas entre os 65 e os 97 anos de idade $(\mathrm{M}=76$ anos; $\mathrm{DP}=7.30$ ), sendo que $66 \%$ dos sujeitos são do sexo feminino e $34 \%$ são do sexo masculino, oriundos do distrito do Porto. A maioria é casada (42\%), sendo os restantes viúvos (38\%).

No que concerne às habilitações académicas, a maioria dos sujeitos tem, no máximo, o $4 .^{\circ}$ ano de escolaridade (55\%). Não obstante, observa-se uma percentagem considerável de participantes com um curso superior (23\%). As profissões dos sujeitos anteriores à sua reforma eram bastante diversificadas, compreendendo quadros superiores, técnicos e profissionais, assim como agricultores e trabalhadores não qualificados. As profissões mais referenciadas foram professor (10\%), doméstica $(9 \%)$, conserveira (3\%), engenheiro (3\%), costureira (3\%), empregada de limpeza (3\%), comercial (2\%) e agricultor (2\%).

\section{RESULTADOS}

Num primeiro momento apresentam-se os resultados descritivos no que concerne à literacia em saúde, seguindo-se os resultados decorrentes das características dos participantes.

\section{Literacia em Saúde: Resultados Descritivos}

As percentagens de respostas corretas são relativamente baixas, variando entre os $18 \%$ (item 6) e os $31 \%$ (item 5). É ainda de evidenciar que a maioria dos participantes elegeu não responder, oscilando as percentagens de não resposta entre os 51\% (item 1) e os $62 \%$ (item 4). De modo análogo, a média foi de 1.36 (DP = 1.96), revelando que os participantes em média acertaram uma resposta em seis, pese embora o valor do desvio-padrão evidenciar bastante variabilidade. Adicionalmente, podemos constatar que uma elevada percentagem de participantes não conseguiu responder ou optou por não responder a nenhuma questão (58\%), enquanto apenas $7 \%$ respondeu corretamente a todas as questões. Verificamos assim existirem níveis de literacia relativamente baixos entre os nossos participantes.

\section{Diferenças nos Níveis de Literacia em Saúde de Acor- do com o Sexo}

De seguida, procede-se à comparação entre os idosos do sexo feminino e do sexo masculino no que diz respeito aos níveis médios de literacia em saúde. Foram realizados testes t para amostras independentes a um nível de significância estatística de 5\%. Para além da significância estatística dos resultados produzidos, recorremos à magnitude do tamanho do efeito verificado. Para tal, foi calculado o coeficiente $\mathrm{d}$ de Cohen, que é baseado nas diferenças estandardizadas de médias, sendo $\mathrm{d}=$ .20 considerado um efeito pequeno ou modesto, $\mathrm{d}=$ .50 um efeito moderado e $\mathrm{d}=.80 \mathrm{um}$ efeito importante (Cohen, 1992). Os resultados obtidos podem ser visualizados no Quadro 1.

Quadro 1 - Testes t para comparação dos resultados obtidos a nível da literacia em saúde nos dois sexos

\begin{tabular}{|l|l|l|l|l|l|l|l|l|l|}
\hline \multicolumn{2}{|c|}{} & \multicolumn{2}{l|}{$\begin{array}{l}\text { Sexo } \\
\text { Masculino }\end{array}$} & \multicolumn{2}{l|}{$\begin{array}{l}\text { Sexo } \\
\text { Feminino }\end{array}$} & \multicolumn{5}{l|}{} \\
\hline & $\mathrm{N}$ & $\mathrm{M}$ & $\mathrm{DP}$ & $\mathrm{M}$ & $\mathrm{DP}$ & $\mathrm{t}$ & $\mathrm{gl}$ & $\mathrm{p}$ & $\mathrm{d}$ \\
\hline $\begin{array}{l}\text { NVS } \\
\begin{array}{l}\text { Literacia } \\
\text { em saúde } \\
\text { (total) }\end{array}\end{array}$ & 433 & 1.66 & 2.06 & 1.21 & 1.91 & 2.18 & 277.09 & .030 & 0.71 \\
\hline $\begin{array}{l}\text { Literacia } \\
\text { em saúde }\end{array}$ & 182 & 3.31 & 1.74 & 3.19 & 1.79 & 0.44 & 180 & .664 & 0.18 \\
\hline
\end{tabular}

${ }^{\star}$ Sujeitos com pelo menos um sucesso $(\mathrm{N}=182)$

No que diz respeito aos níveis médios de literacia em saúde, verifica-se que os participantes do sexo masculino obtiveram notas médias mais elevadas do que os participantes do sexo feminino, $t(277.09)=2.18, \mathrm{p}<$ .05. Esta diferença foi considerada estatisticamente significativa e o valor do coeficiente d de Cohen de .17 indica um efeito pequeno. Estas diferenças desaparecem, no entanto, quando excluímos os sujeitos com total insucesso na prova, que representam 58\% da amostra. Para os 108 sujeitos do sexo feminino e 74 sujeitos do sexo masculino que têm pelo menos um sucesso no teste, as médias são próximas do valor médio de 3, respetivamente, 3.19 e 3.31, não sendo a diferença estatisticamente significativa, $\mathrm{t}(180)=0.44$, ns. De facto, as diferenças a nível do género parecem residir na proporção de homens e mulheres com total insucesso no teste: $50 \%$ nos primeiros e $62 \%$ nas segundas (cf. Figura 1). Um teste de qui-quadrado parece indicar que estas proporções são estatisticamente significativas, $\chi 2(1)=5.86$, $\mathrm{p}=.016$, pelo que se pode inferir que as mulheres têm maior probabilidade de não responderem corretamente a nenhum item no teste. 
Figura 1 - Percentagem de participantes de acordo com o número de respostas corretas no NVS relativamente ao género $(\mathrm{N}=433)$

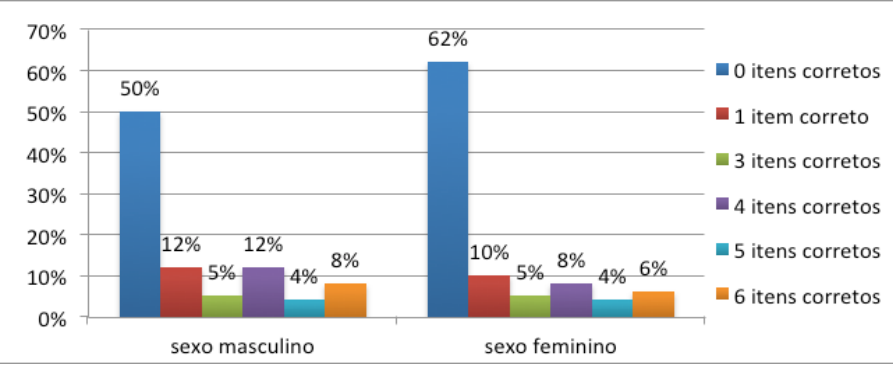

\section{Associações entre os Níveis de Literacia em Saúde e a Idade}

De modo a conhecer a associação entre os níveis de literacia em saúde e a idade, foi calculada a correlação de Pearson a um nível de significância estatística de 5\%. O coeficiente de correlação foi interpretado em termos do tamanho do efeito, de acordo com as convenções de Cohen (1992): um $r$ de .10 é considerado pequeno (associação fraca), um $\mathrm{r}$ de .30 é considerado médio (associação moderada) e um $r$ de .50 é considerado grande (associação forte). A associação encontrada foi negativa, média $r=-.34$, o que indica que os valores das duas variáveis tendem a variar em sentido inverso, ou seja, os níveis de literacia tendem a ser mais baixos com o avanço da idade.

\section{Diferenças nos Níveis de Literacia em Saúde de acor- do com o Nível de Escolaridade}

Com o intuito de examinar o efeito do nível de escolaridade dos inquiridos nos níveis de literacia em saúde, tendo verificado que os dados não cumpriam os requisitos exigidos pela análise de variância, nomeadamente no que concerne à homogeneidade de variância, recorreu-se às análises não paramétricas, especificamente ao teste de Kruskal-Wallis, a um nível de significância estatística .05. Para identificar diferenças específicas entre os grupos, conduzimos testes de Mann-Whitney, a um nível de significância estatística .0083 , após a correção de Bonferroni. Recorremos aos quatro níveis criados para descrever a escolaridade dos inquiridos (nível $1=$ até ao $4 .^{\circ}$ ano de escolaridade; nível $2=$ entre o $5 .^{\circ} \mathrm{e}$ 9. ${ }^{\circ}$ anos de escolaridade; nível 3 = ensino secundário e nível 4 = ensino superior). Os resultados obtidos podem ser visualizados no Quadro 2.
Quadro 2 - Comparação dos resultados obtidos no NVS de acordo com o nível de escolaridade

\begin{tabular}{|l|l|l|l|l|l|l|l|l|l|}
\hline & \multicolumn{2}{|l|}{$\begin{array}{l}1 .{ }^{\circ} \mathrm{CEB} \\
(\mathrm{n}=235)\end{array}$} & \multicolumn{2}{|l|}{$\begin{array}{l}2 . \% 3 .^{\circ} \mathrm{CEB} \\
(\mathrm{n}=60)\end{array}$} & \multicolumn{2}{l|}{$\begin{array}{l}\text { Ensino } \\
\text { Secundário } \\
(\mathrm{n}=34)\end{array}$} & \multicolumn{2}{l|}{$\begin{array}{l}\text { Ens. Uni- } \\
\text { versitário } \\
(\mathrm{n}=96)\end{array}$} & \\
\hline & $\mathrm{M}$ & $\mathrm{DP}$ & $\mathrm{M}$ & $\mathrm{DP}$ & $\mathrm{M}$ & $\mathrm{DP}$ & $\mathrm{M}$ & $\mathrm{DP}$ & \\
\hline $\begin{array}{l}\text { Literacia } \\
\text { em saúde }\end{array}$ & 0.35 & 0.86 & 1.13 & 1.60 & 2.82 & 1.88 & 3.48 & 2.20 & $\begin{array}{l}\mathrm{G} 4=\mathrm{G} 3> \\
\mathrm{G} 2>\mathrm{G} 1\end{array}$ \\
\hline
\end{tabular}

Como se pode constatar, foi encontrado um efeito estatisticamente significativo, indiciando existirem diferenças entre os vários grupos de sujeitos, $\mathrm{H}(3)=175.22$, $\mathrm{p}<.001$. As análises Mann-Whitney permitiram verificar que os indivíduos com o 1. ${ }^{\circ} \mathrm{CEB}$ (até 4 anos de escolaridade) apresentaram resultados mais baixos do que todos os outros grupos, $\mathrm{U}=5171.50, \mathrm{p}<.001, \mathrm{U}$ $=1184.00, \mathrm{p}<.001, \mathrm{U}=2864.50, \mathrm{p}<.001 . \mathrm{Da}$ mesma forma, os indivíduos com o $2 .^{\circ}$ ou $3 .^{\circ} \mathrm{CEB}$ apresentaram resultados mais baixos do que os grupos de sujeitos com o ensino secundário e o ensino universitário, respetivamente, $\mathrm{U}=520.00, \mathrm{p}<.001$ e $\mathrm{U}=1212.50, \mathrm{p}$ $<.001$. Não se verificaram diferenças nas médias entre os indivíduos com ensino secundário e ensino superior, $\mathrm{U}=1292.50, \mathrm{p}=.067$. Assim, e como seria de esperar, verifica-se que o nível de escolaridade tem um papel determinante nos níveis médios de literacia em saúde.

\section{Diferenças nos Níveis de Literacia em Saúde de acor- do com o Estado Civil}

De modo a analisar o efeito do estado civil nos níveis de literacia em saúde, consideraram-se as diferenças entre os indivíduos casados $(\mathrm{n}=185)$ e os viúvos $(\mathrm{n}=166)$. Os idosos solteiros $(\mathrm{n}=37)$, em união de facto $(\mathrm{n}=$ $7)$, separados $(n=8)$ e divorciados $(n=29)$ foram excluídos das análises dado o número reduzido com que surgiram nesta amostra. Foram realizados testes $t$ para amostras independentes a um nível de significância estatística de $5 \%$. Os resultados obtidos podem ser visualizados no Quadro 3.

Quadro 3 - Testes t para comparação dos resultados obtidos a nível da literacia em saúde de acordo com o estado civil

\begin{tabular}{|l|l|l|l|l|l|l|l|l|}
\hline & \multicolumn{2}{|l|}{$\begin{array}{l}\text { Casados } \\
(\mathrm{n}=185)\end{array}$} & \multicolumn{2}{l|}{$\begin{array}{l}\text { Viúvos } \\
(\mathrm{n}=166)\end{array}$} & \multicolumn{4}{l|}{} \\
\hline & $\mathrm{M}$ & $\mathrm{DP}$ & $\mathrm{M}$ & $\mathrm{DP}$ & $\mathrm{t}$ & $\mathrm{gl}$ & $\mathrm{p}$ & $\mathrm{d}$ \\
\hline $\begin{array}{l}\text { Literacia } \\
\text { em saúde }\end{array}$ & 1.98 & 2.21 & 0.70 & 1.43 & 6.49 & 319.3 & .000 & 0.51 \\
\hline
\end{tabular}


Os indivíduos casados tendem a obter valores médios de literacia em saúde mais elevados do que os viúvos, $\mathrm{t}(319.3)=6.49, \mathrm{p}<.05$. O valor do coeficiente $\mathrm{d}$ de Cohen de 0.68 indica um efeito moderado. Contudo, os grupos diferem também relativamente à idade, apresentando o grupo dos indivíduos viúvos uma média de idade mais elevada, $\mathrm{M}=79.86, \mathrm{DP}=6.23$, quando comparados com os sujeitos casados, $\mathrm{M}=73.77, \mathrm{DP}=$ $6.80, \mathrm{t}(349)=-8.74, \mathrm{p}<.05$. Assim, procedemos novamente à comparação entre os dois grupos, controlando a idade. Os resultados mostraram que as diferenças entre os grupos se mantinham, mesmo quando controlada a idade, $\mathrm{F}(1,348)=16.30, \mathrm{p}<.05$. Assim, o estado civil parece desempenhar um papel relevante nos níveis de literacia em saúde entre os sujeitos destes dois grupos.

\section{DISCUSSÃO}

Da análise dos resultados podemos referir que o grau de literacia em saúde obtido foi de $1.37 \pm 1.98$, o que evidencia uma inadequada literacia em saúde dos sujeitos participantes neste estudo. Estes resultados são corroborados por vários estudos (Speros, 2005; Sudore et al., 2006), legitimando a urgência de um trabalho de investigação e de intervenção em profundidade.

No que diz respeito às variáveis sociodemográficas estudadas, verificámos diferenças muito significativas de acordo com a idade, as habilitações literárias e o sexo. As pessoas que têm menos idade assim como as que têm um maior grau de escolaridade apresentam mais respostas certas, o que se assemelha aos resultados obtidos noutras pesquisas internacionais (Baker et al., 2000; Bennett et al., 2009). No que concerne ao sexo, os participantes do sexo masculino obtiveram notas médias mais altas quando comparados com os do sexo feminino. Nos estudos internacionais desenvolvidos, como referimos anteriormente, não há concordância nos resultados obtidos. Naqueles em que os homens apresentavam uma maior literacia em saúde, os autores associavam-na a uma maior escolaridade das pessoas do sexo masculino neste grupo etário (Jovic-Vranes et al., 2009); já naqueles em que as mulheres apresentavam resultados superiores, levantou-se como hipótese explicativa o facto de as mesmas serem mais capazes de prestarem cuidados e de cuidarem das pessoas doentes da sua família, tendo, por isso mesmo, maior contacto com os contextos de saúde (Sudore et al., 2006).

Por fim, observou-se que os indivíduos casados tendem a obter valores médios de literacia em saúde mais elevados do que os viúvos. São, assim, necessários estudos, nacionais e internacionais, que estudem o impacte desta variável no nível da literacia em saúde.

\section{CONCLUSÃO}

Os resultados indicam que a grande maioria dos sujeitos $(80 \%)$ evidencia um nível de literacia em saúde baixo, o que significa que apenas $20 \%$ dos inquiridos serão capazes de interpretar e usar a informação escrita relacionada com a saúde de forma eficaz. As análises conduzidas apontam para um efeito notável de algumas variáveis demográficas nos valores médios obtidos no teste de literacia em saúde, especificamente, sexo, idade, habilitações literárias e estado civil.

\section{IMPLICAÇÕES PARA A PRÁTICA CLÍNICA}

$\mathrm{Na}$ senda dos resultados obtidos, neste e em outros estudos, urge potenciar a literacia em saúde na população idosa, e, dentro desta, junto dos grupos de maior vulnerabilidade. Como recomenda a WHO (2013), os políticos, os profissionais, a sociedade civil e o setor privado podem cooperar no sentido de promoverem e enfrentarem os desafios relativos à literacia em saúde. Destacamos, entre estes, os profissionais de saúde e de intervenção psicossocial, já que estes são profissionais de proximidade, inseridos em Organizações de saúde e de apoio à terceira idade, com os quais os idosos estabelecem uma relação privilegiada. Além de apelarem a uma participação ativa dos sujeitos no desenvolvimento de atitudes saudáveis e na prevenção e/ou resolução de problemas de saúde, podem e devem disponibilizar informações, pertinentes e claras, e um acompanhamento de maior proximidade de acordo com as características e necessidades singulares de cada idoso, potenciando a sua literacia em saúde e, consequentemente, a sua qualidade de vida.

\section{REFERÊNCIAS}

Baker, D. W., Gazmararian, J. A., Sudano J., \& Patterson, M. (2000). The association between age and health literacy among elderly persons. The Journals of Gerontology, Series B: Psychological Sciences and Social Sciences, 55(6), 368-374. doi: 10.1093/gernb/55.6.S368

Bennett, I., Chen, J., Soroui, J., \& White, S. (2009). The contribution of health literacy to disparities in selfrated health status and preventive health behaviors in older adults. Annals of Family Medicine, 7(3), 204-211. doi: 10.1370/afm.940 
Jovic-Vranes, A., Bjegovic-Mikanovic, V., \& Marinkovic J. (2009). Functional health literacy among primary health-care patients: data from the Belgrade pilot study. Journal of Public Health (Oxford), 31(4), 490-495. doi: 10.1093/pubmed/fdp049

Kim, S. (2009). Health literacy and functional health status in Korean older adults. Journal of Clinical Nursing, 18, 2337-2343. doi: 10.1111/j.1365-2702.2008.02739.x. Manafo, E., \& Wong, S. (2012). Health literacy programs forolderadults:asystematicliterature review.HealthEducation Research, 27(6), 947-960.doi: 10.1093/her/cys067

Schillinger, D., Grumbach, K., Piette, W. F., Osmond, D., Daher, C., Palacios, J., Sullivan, G. D., \& Bindman, A. B. (2002). Association of health literacy with diabetes outcomes. JAMA, 288, 475-482. doi:10.1001/ jama.288.4.475

Speros, C. (2005). Health literacy: Concept analysis. Journal of Advanced Nursing, 50(6), 633-640. doi: 10.1111/j.1365-2648.2005.03448.x

Sudore, R. L., Mehta, K. M., Simonsick, E. M., Harris, T. B., Newman, A. B., Satterfield, S., Rosano, C., Rooks, R. N., Rubin, S. N., Ayonayon, H. A., \& Yaffe, K. (2006). Limited literacy in older people and disparities in health and healthcare access. Journal of the American Geriatrics Society, 54(5), 770-776. doi: 10.1111/j.15325415.2006.00691.x
Toçi, E., Burazeri, G., Sorensen, Jerliu, N., Ramadani, N., Roshi, E., \& Brand, H. (2013). Health literacy and socioeconomic characteristics among older people in transitional Kosovo. British Journal of Medicine \& Medical Research, 3(4), 1646-1658. doi: 10.9734/ BJMMR/2013/3972

Von Wagner, C., Knight, K., Steptoe, A., \& Wardle, J. (2007). Functional health literacy and health-promoting behaviour in a national sample of British adults. Journal of Epidemiology \& Community Health, 61(12), 1086-90. doi: 10.1136/jech.2006.053967

Weiss, B. D., Mays, M. Z., Martz, W., Castro, K. M., DeWalt, D. A, Pignone, M. P., \& Mockbee, J. (2005). Quick assessment of literacy in primary care: the newest vital sign. Annals of Family Medicine, 3(6), 514-22. doi: 10.1370/afm.405

Wolf, M., Gazmararian, J., \& Baker, D. (2005). Health literacy and functional health status among older adults. Archives of Internal Medicine, 165, 1946-1952. doi: 10.1001/archinte.165.17.1946

World Health Organization (2013). Health literacy. The solid facts. Copenhagen: WHO Regional Office for Europe.

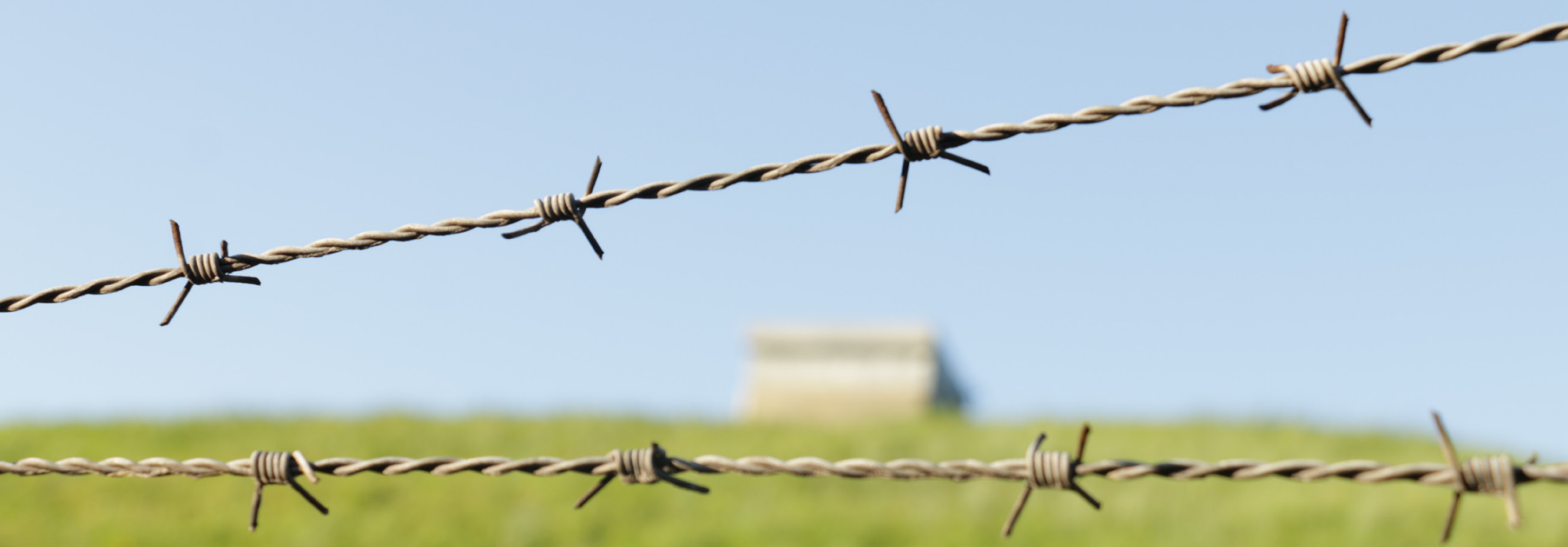

